\title{
Strong Interactions in the Regge Limit and Infrared Region
}

\author{
Carlos Contreras ${ }^{1 *}$, Jochen Bartels ${ }^{2}$ and Gian Paolo Vacca ${ }^{3}$ \\ ${ }^{1}$ Departament of Physics, Santa Maria University, Avda España 1680, Valparaiso, Chile \\ ${ }^{2}$ II Institut für Theoretishe Physik, Universität Hamburg, D-22761 Hamburg. Germany \\ ${ }^{3}$ INFN Sezione di Bologna, DIFA, via Irnerio 46, I-40126 Bologna, Italia
}

\begin{abstract}
In this talk, we encode the perturbative BFKL leading logarithmic resummation, relevant for the Regge limit behavior of QCD scattering amplitudes, in the IR regulated effective action, which satisfies exact functional renormalization group equations. The goal is to use this framework to study, in the high-energy limit and at larger transverse distances the transition to a much simpler effective local Reggeon field theory, whose critical properties were recently investigated in the same framework. We perform a numerical analysis of the spectrum of the BFKL Pomeron by the introduction of a Wilsonian infrared regulator to understand the properties of the leading poles (Pomeron states) contributing to the high-energy scattering
\end{abstract}

\section{Introduction}

Understanding the physics of strong interactions (QCD) through the study of scattering processes in different kinematical regimes is still a challenging task. Particularly interesting is the kinematic region of very high energies and finite momentum transfers (Regge limit): in contrast to those high-energy limits where collinear factorization holds and provides a clean separation between perturbative and nonperturbative QCD, the Regge limit has no such strict separation. It is therefore tempting to investigate, in the Regge limit, the transition from the perturbative QCD regime (controlled by small distances) to the region of large distances, for which still very little can be derived from a fundamental QCD description. In papers [13], we propose an approach to find a way that interpolates between the perturbative QCD Pomeron (BFKL Pomeron) and the nonperturbative soft Pomeron which describes elastic proton-proton scattering at high energies. In transverse space, the BFKL Pomeron and the soft Pomeron, are probing different regions: the BFKL Pomeron describes processes which are dominated by small transverse distances (large transverse momenta), whereas the soft Pomeron is sensitive to transverse distances of the order of or larger than the proton radius. Wilsonian exact renormalization group techniques can be used both on the perturbative (UV) region and in the nonperturbative (IR) region. In particular, we have found it convenient to study the flow of an effective average whose RG-flow is described by the Wetterich equation $[4,5]$.

The long distance analysis (IR region) can be found in $[1,2]$ where we have studied the flow of the Reggeon Field Theory (RFT) for a single Pomeron field and for a Pomeron coupled to an Odderon field, in particular the critical universal properties of these two. These

* carlos.contreras@usm.cl 
theories were formulated before QCD [6-9], expecting that they could describe the nonperturbative large distance behavior of the strong interactions. As a results, critical solution has been found, and scaling laws for the asymptotic scattering amplitude have been derived. In our recent analysis, we include a general search for IR fixed points (i.e. in the presence of all possible Pomeron self-couplings), the behavior also near the fixed points, i.e. the high energy behavior of scattering processes at large but finite energies. Finally, we made an analysis of global flows within a RFT description and we have included the Odderon.

This review paper summarizes our recent study in the UV region [3]: we start from high energy QCD, as formulated in Lipatov's effective action [10], generically describing the BFKL Physics regime. Here the most important degree of freedom is the reggeized gluon, built from gluon exchanges at high energies. The perturbative BFKL Pomeron [11-14] then appears as a composite state of two reggeized gluons in color singlet. In leading order, it has a continuous spectrum for the variable angular momentum which is dual to the rapidity. The presence of vertices, local in rapidity, but nonlocal in the transverse space, maps QCD in the Regge limit into an effective $2+1$ dimensional QFT of reggeized gluons. Considering their composite states which couple directly to external asymptotic states (i.e. hadrons) through impact factors, it is tempting to switch, starting from the reggeized gluons, to another effective description in terms of colorless composite states of reggeized gluons. As an example, with such a 'switch', the 2 to 4 reggeized gluon vertex $[15,19]$ induces a perturbative triple Pomeron vertex, so that the BFKL Pomeron interacts with itself. The extension to other vertices, which describe the interactions of the BFKL Pomeron with the perturbative Odderon can be found in ref. [20,21].

It is known that in NLO [22], when the QCD coupling becomes momentum dependent, some infrared cutoff has to be introduced which, strictly speaking, already goes beyond perturbation theory. The BFKL Pomeron spectrum then becomes discrete, even at relatively short distances. This corresponds to the appearance of bound state Regge poles (Pomeron states), and it needs to be investigated which of them and in what way they will contribute to the scattering processes at large distances and large rapidities. In order to study the interactions of these Regge poles and in order to be able to move to larger distances it will be convenient to switch to bound state fields, to consider the local approximation and to make use of the well-known formalism of RFT. This step will make it much easier to include pomeron interactions which in the full QCD description are described by non local vertex functions. In the field theory based upon reggeized gluons, these vertex functions would lead to an extremely hard problem to solve, but we expect to be irrelevant at large distances. We stress that both frameworks, BFKL physics and RFT, live in $2+1$ dimensions, transverse momenta (transverse distances) and rapidity (angular momentum).

In order to study the transition from the UV to the IR region it is necessary to have a description which allows to trace the transmutation of the degrees of freedom at the different relevant scales. This can be controlled by introducing an infrared cutoff for the transverse momentum: initially (in the UV region) the momentum cutoff has to be large to justify the applicability of perturbation theory for QCD in the Regge limit. Lowering this cutoff brings us into the infrared, nonperturbative region: first still for the QCD description, then for the local RFT (with an IR cutoff). The main task then is to bring these two last pictures together: the bound states of the BFKL Pomeron define Pomeron fields, which serve as an input into RFT. As a theoretical framework, we choose the IR regulated effective action, satisfying exact renormalization group equations, as the way to encode the different dynamical regimes. We begin with the BFKL equation and introduce an infrared regulator as suggested by the $\mathrm{RG}$ equations. With this regulator we derive a differential equation for the BFKL Pomeron Green's function with respect to the IR cutoff parameter $\mathrm{k}$. This equation has the form of the infrared evolution equations; moreover, it is nonlinear and characterized by a two loop structure. In the next step we have to find an effective field theory which can be used to derive 
the BFKL Pomeron. We start from Lipatov's effective action and formulate an effective field theory for the 1PI vertices associated to composite states of two reggeized gluons. This field theory contains separate fields for the reggeized gluon and for elementary gluons. Introducing the infrared regulator we derive, for the effective field theory of the reggeized gluon fields, exact functional RG flow equations. By construction the solution of the flow equation can be obtained from the knowledge of the IR regulated BFKL kernel and Green's function.

In the second part of this presentation, we perform a numerical analysis of the BFKL Pomeron with our infrared regulator. In our analysis, we will not yet make use of the functional RG equations derived before, but, instead, find explicit solutions of the BFKL equation for a few fixed values of the infrared cutoff. To make contact with a previous analysis [23-29] of the BFKL spectrum we begin with a numerical study of the BFKL Pomeron with a gluon mass regulator. We then compute the BFKL spectrum using our infrared regulator which, in a future part of our analysis, will allow to use the BFKL Pomeron as an input to the RG flow. We compute energy eigenvalues, in particular intercepts and $q^{2}$ slopes of the trajectory functions and eigenfunctions of the BFKL kernel: these parameters define a set of Pomeron states.

This paper is organized as follows. In section 2 we present the leading order BFKL equation and introduce the infrared regulator. In Section 3 we discuss the nonlinear differential equation for the dependence upon the infrared regulator. Sections 4 describe our numerical studies. Finally, the summary and outlook on the following steps of our program is given in Sec. 5 .

\section{BFKL Equation with regulator}

In the UV region, we start from QCD perturbation theory and the massless color singlet BFKL (see ref. [11-14]) in the leading multi-Regge kinematics (MRK). The BFKL Pomeron Green's function $G\left(\boldsymbol{q}^{\prime}, \boldsymbol{q}-\boldsymbol{q}^{\prime} ; \boldsymbol{q}^{\prime \prime}, \boldsymbol{q}-\boldsymbol{q}^{\prime \prime} \mid \omega\right)$ which is obtained as an infinite sum of ladder diagrams, satisfies the integral equation:

$$
\begin{gathered}
G_{B F K L}\left(\boldsymbol{q}^{\prime}, \boldsymbol{q}-\boldsymbol{q}^{\prime} ; \boldsymbol{q}^{\prime \prime}, \boldsymbol{q}-\boldsymbol{q}^{\prime \prime} \mid \omega\right)=K_{B F K L}\left(\boldsymbol{q}^{\prime}, \boldsymbol{q}-\boldsymbol{q}^{\prime} ; \boldsymbol{q}^{\prime \prime}, \boldsymbol{q}-\boldsymbol{q}^{\prime \prime}\right)+ \\
\int d^{2} \boldsymbol{k} \frac{K_{B F K L}\left(\boldsymbol{q}, \boldsymbol{q}-\boldsymbol{q}^{\prime} ; \boldsymbol{k}, \boldsymbol{q}-\boldsymbol{k}\right)}{\omega-\omega_{g}(\boldsymbol{k})-\omega_{g}(\boldsymbol{q}-\boldsymbol{k})} \frac{G_{B F K L}\left(\boldsymbol{k}, \boldsymbol{q}-\boldsymbol{k} ; \boldsymbol{q}^{\prime}, \boldsymbol{q}-\boldsymbol{q}^{\prime \prime} \mid \omega\right)}{\boldsymbol{q}^{\prime 2}\left(\boldsymbol{q}-\boldsymbol{q}^{\prime}\right)^{2}}
\end{gathered}
$$

where $\omega$ is the dual variable to the rapidity and $\boldsymbol{q}^{\prime}$ s are two dimensional transverse momentum variables. This equation can be represented diagrammatically as in Fig.1 and look like as a Bethe-Saltpeter equation. One can find that the expression of the LO BFKL Kernel $K_{B F K L}$ and for $\omega_{g}(\boldsymbol{k})$ the gluon trajectory are given by:

$$
\begin{gathered}
K_{B F K L}\left(\boldsymbol{q}^{\prime}, \boldsymbol{q}-\boldsymbol{q}^{\prime} ; \boldsymbol{q}^{\prime \prime}, \boldsymbol{q}-\boldsymbol{q}^{\prime \prime}\right)=\frac{\bar{\alpha}}{2 \pi}\left[-\boldsymbol{q}^{2}+\frac{\boldsymbol{q}^{\prime \prime 2}\left(\boldsymbol{q}-\boldsymbol{q}^{\prime}\right)^{2}}{\left(\boldsymbol{q}^{\prime \prime}-\boldsymbol{q}^{\prime}\right)^{2}}+\frac{\boldsymbol{q}^{\prime 2}\left(\boldsymbol{q}-\boldsymbol{q}^{\prime \prime}\right)^{2}}{\left(\boldsymbol{q}^{\prime \prime}-\boldsymbol{q}^{\prime}\right)^{2}}\right] \\
\omega_{g}(\boldsymbol{q})=-q^{2} \frac{\bar{\alpha}}{2 \pi} \int d^{2} \boldsymbol{k} \frac{1}{\boldsymbol{k}^{\mathbf{2}}(\boldsymbol{q}-\boldsymbol{k})^{\mathbf{2}}}
\end{gathered}
$$

where $\bar{\alpha}=N_{C} \alpha / \pi$. 


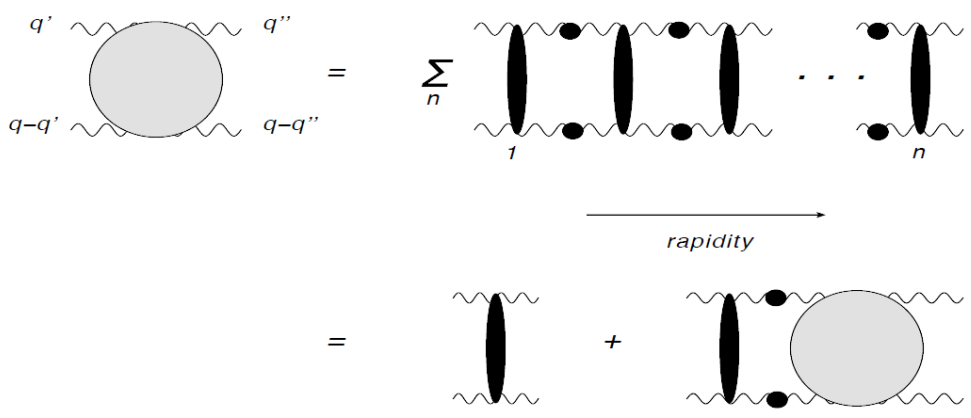

Fig. 1. BFKL Ladder. Wavy line are reggeized gluon and internal straight-line elementary gluons

In order to arrive to an IR safe version of the BFKL equation we have to introduce some infrared cutoff, which, strictly speaking, already goes beyond perturbation and we have to symmetrize the kernel:

$$
K\left(\boldsymbol{q}^{\prime}, \boldsymbol{q}-\boldsymbol{q}^{\prime} ; \boldsymbol{q}^{\prime \prime}, \boldsymbol{q}-\boldsymbol{q}^{\prime \prime}\right)=\frac{K_{B F K L}\left(\boldsymbol{q}^{\prime}, \boldsymbol{q}-\boldsymbol{q}^{\prime} ; \boldsymbol{q}^{\prime \prime}, \boldsymbol{q}-\boldsymbol{q}^{\prime \prime}\right)}{\sqrt{\boldsymbol{q}^{\prime 2}\left(\boldsymbol{q}-\boldsymbol{q}^{\prime \prime}\right)^{2}} \sqrt{\boldsymbol{q}^{\prime \prime 2}\left(\boldsymbol{q}-\boldsymbol{q}^{\prime \prime}\right)^{2}}}
$$

Finally, the BFKL which contains the real part (gluon emission) and the virtual part (gluon trajectory) is

$$
\widetilde{K}\left(\boldsymbol{q}^{\prime}, \boldsymbol{q}-\boldsymbol{q}^{\prime} ; \boldsymbol{q}^{\prime \prime}, \boldsymbol{q}-\boldsymbol{q}^{\prime \prime}\right)=K+\delta^{2}\left(\boldsymbol{q}^{\prime}-\boldsymbol{q}^{\prime \prime}\right)\left[\omega_{g}\left(\boldsymbol{q}^{\prime}\right)+\omega_{g}\left(\boldsymbol{q}-\boldsymbol{q}^{\prime}\right)\right] .
$$

This LO BFKL equation is for fixed coupling constant $\bar{\alpha}$. To get closer to realistic physics we need to include the running coupling $\bar{\alpha}(q)$, substituting the leading order of the $\bar{\alpha}(q)$ at the fixed constant value.

As we know the BFKL equation correspond to an effective Bethe Salpeter equation. Then we can start to study the spectral properties of the BFKL Green's function. In this approach the Pomeron appears as a bound state of two reggeized gluon. Introducing an IR regulator, (as for example the functional RG cutoff $\mathrm{k}: R_{k}\left(q^{2}\right)$ ) one can show that the spectrum of the BFKL kernel $\widetilde{K}$ consists of a discrete and continuum part. Then we can write

$$
G_{B F K L}=\frac{1}{\omega-\widetilde{K}}=\sum_{n} \frac{f_{n, k}\left(q^{\prime}, q-q^{\prime}\right) f_{n, k}^{*}\left(q^{\prime \prime}, q-q^{\prime \prime}\right)}{\omega-\omega_{n, k}}+\text { continuos part }
$$

where the $f_{n, k}$ are the eigenfunctions of the BFKL Kernel, which we will compute numerically in the second part of this paper for ERG IR regulators. This regulator is different from others that have been used before: Higgs mass [22-26], IR Boundary Conditions [2729].

In order to investigate the dependence of the previous result on the regulator, one can derive a differential equation for the BFKL Pomeron Green function with respect to the IR cutoff parameter. We will obtain a nonlinear equation which is of the same form as obtained by Lipatov and Kirschner [31-32]. In the next section we will show that, with this regulator, the BFKL Pomeron can be formulated using the ERG approach which is related with the flow equation a la Wetterich.

To implement the Wilsonian RG approach, we introduce the momentum regulator for the elementary gluon propagator inside the BFKL kernel and the gluon trajectory:

$$
\frac{1}{\boldsymbol{q}^{2}} \rightarrow \frac{1}{\left[\boldsymbol{q}^{2}+R_{k}\left(\boldsymbol{q}^{2}\right)\right]}
$$

This regulator is such that the propagation of the quantum fluctuations is inhibited for the $q^{2} \ll k^{2}$, but is unaltered in the UV region. In this analysis, we will use the optimized Litim regulator [33] defined by:

$$
R_{k}\left(q^{2}\right)=\left(k^{2}-q^{2}\right) \boldsymbol{\Theta}\left(k^{2}-q^{2}\right),
$$


and the regulated gluon trajectory function (5) is given by:

$$
\omega_{g, k}\left(\boldsymbol{q}^{2}\right)=-q^{2} \frac{\bar{\alpha}}{4 \pi} \int d^{2} \boldsymbol{k}^{\prime} \frac{1}{\left[\boldsymbol{k}^{\prime 2}+R_{k}\left(\boldsymbol{k}^{\prime 2}\right)\right]\left[\left(\boldsymbol{q}-\boldsymbol{k}^{\prime}\right)^{2}+R_{k}\left(\left(\boldsymbol{q}-\boldsymbol{k}^{\prime}\right)^{2}\right)\right]} .
$$

In similar way, we need to rewrite the BFKL kernel and the Green's functions $G_{B F K L}$.

\section{RG flow equation}

Before to perform a numerical analysis, let us study the flow equation in terms of the cutoff. In order to obtain the flow equation, we have two option. Starting from the regulated Green's functions $G_{B F K L, k}$ we can take the derivative with respect to $\tau=\ln k / k_{0}$. Diagrammatically, we can start from the Fig 1. which describe the integral equation of the Green's function, and the derivative with respect to the IR regulator (see Fig. 2) acts on the internal lines for the elementary gluons (straight line) and reggeized gluons (wavy line). Finally, (for more details see ref. [3]) after a straightforward calculation, we could obtain a nonlinear structure of this equation which is the same as in the IR evolution equation of Lipatov and Kirschner. We would like to show that the same equation for $\tau$-derivative can be obtained from the RG flow equation in the MRK.

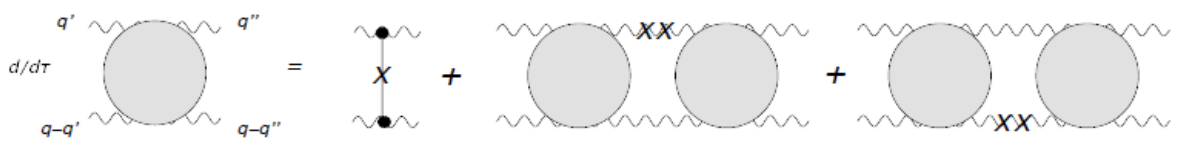

Fig. 2. k-derivative of the BFKL ladder. The crosses on internal lines denote the $\mathrm{k}$ derivative. The dots denote the momentum factors of the vertices.
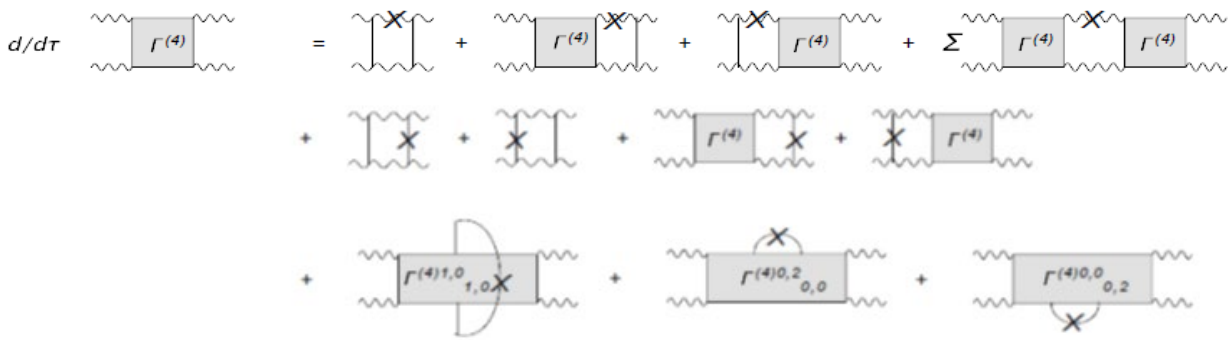

Fig. 3. Diagrammatic representation for the RG flow equation for the 1 PI 4-point function. The crosses mark the derivative of the momentum regulator and the internal wave-line describe the regulated propagator.

To this end, we have to study the flow equation derived by the ERG Wetterich's equation:

$$
k \frac{\partial \Gamma_{k}}{\partial k}=\frac{1}{2} \operatorname{Tr}\left[\left(\Gamma_{k}^{(2)}+\mathrm{R}_{k}\right)^{-1} k \frac{\partial \mathrm{R}_{k}}{\partial k}\right] .
$$

However, we need a suitable truncation of the effective average action $\Gamma_{\mathrm{k}}$. In this case, we start with the effective Lipatov's field theory which describe the Regge limit of QCD and introduce the reggeized gluon [10]. With this effective field theory of reggeized gluons which describes the leading order BFKL Green's function we can discuss how to make a transition to bound state Pomeron fields. It is not difficult to show that the flow equation form an infinite tower of coupled $n$-point $\Gamma_{k}^{(n)}$ functions. We then make use of a special feature of this field theory and derive, for the BFKL Green's function (BFKL 4-point function), a nonlinear equation, which coincides with the $\tau$ - derived equation

In order to find the connection with the previous result, we shall consider (see Fig 3) the vertices with 4 reggeized gluons and study the flow equation for the 1PI 4-points function 
$\Gamma_{k}{ }^{(4)}$. The flow equation for the 1PI 4-point function of the process: two reggeized gluons to two reggeized (i.e. the BFKL Green's function) appears as part of an infinite set of coupled flow equations which also contain higher order processes, e. g. two reggeized gluons plus elementary gluon to two reggeized plus elementary gluon. It is straightforward making use of special features of this field theory: in particular constraints derived from the MRK and that the gluons do not propagate in rapidity to derive, from the functional $\mathrm{RG}$ flow equations, the same nonlinear differential equation for the BFKL Pomeron Green's function which was obtained before. In other words, we show, for the BFKL Green's function of two incoming and two outgoing reggeized gluons, that the infinite set of functional RG flow equations set is equivalent to a single nonlinear differential equation. For our analysis of the BFKL Pomeron, we only need the lowest nonlinear equation.

\section{Numerical Analysis}

In this section, we shall perform a carefully numerical analysis of the IR modified BFKL Kernel: first, introducing a simple mass regulator (we partly follows the papers [24]) and then a Wilsonian optimized regulator. We begin with the fixed QCD coupling, and in a second step, we also consider the effect of the running gauge coupling. Our focus is on the spectrum of the integral kernel: eigenvalues, eigenfunctions. What is new in our analysis is the $q^{2}-$ slopes analysis of the discrete Pomerons states. It is for a discrete spectrum that one can find new effective degrees of freedom (the Pomeron appears as a bound state of two reggeized gluon) and make a link at large distances with the local Pomeron fields of a RFT. Therefore, we shall look for evidence of such a case. This result is relevant, since in the next (future) step, these BFKL Pomerons and its spectrum will be used to define the input to the exact RG (ERG) flow equations. To this end, it is necessary to find a formulation of the BFKL Pomeron which is compatible with the ERG flow equations.

\subsection{Eigenvalues and eigenfunctions in the forward direction for mass regulator}

We begin with the Higgs mass regulated BFKL Pomeron with fixed coupling. Let us to introduce: $\boldsymbol{q}_{1}=\boldsymbol{q} / 2+\boldsymbol{k} ; \boldsymbol{q}_{2}=\boldsymbol{q} / 2-\boldsymbol{k}$; and $\boldsymbol{q}_{1}^{\prime}=\boldsymbol{q} / 2+\boldsymbol{k}^{\prime} ; \boldsymbol{q}_{2}^{\prime}=\boldsymbol{q} / 2-\boldsymbol{k}^{\prime}$, and we have to consider the kernel:

$$
\widetilde{K}\left(\boldsymbol{q}, \boldsymbol{k}, \boldsymbol{k}^{\prime}\right)=K\left(\boldsymbol{q}, \boldsymbol{k}, \boldsymbol{k}^{\prime}\right)+\delta^{2}\left(\boldsymbol{k}-\boldsymbol{k}^{\prime}\right)\left[\omega_{g}\left(\boldsymbol{q}_{1}^{2}\right)+\omega_{g}\left(\boldsymbol{q}_{2}^{2}\right)\right]
$$

where the gluon trajectory has the form

$$
\omega_{g}\left(k^{2}\right)=-\frac{\bar{\alpha}}{2 \pi} \int d^{2} \boldsymbol{k}^{\prime} \frac{\boldsymbol{k}^{2}+m^{2}}{\left(\boldsymbol{k}^{\prime 2}+m^{2}\right)\left(\boldsymbol{k}^{\prime 2}+\left(\boldsymbol{k}-\boldsymbol{k}^{\prime}\right)^{2}+2 m^{2}\right)}
$$

and the symmetrized kernel:

$$
\begin{gathered}
\frac{2 \pi}{\bar{\alpha}} K\left(\boldsymbol{q}, \boldsymbol{k}, \boldsymbol{k}^{\prime}\right)=\sqrt{\frac{q_{1}^{2}+m^{2}}{q_{2}^{2}+m^{2}}} \frac{1}{\left(\left(\boldsymbol{k}-\boldsymbol{k}^{\prime}\right)^{2}+m^{2}\right)} \sqrt{\frac{q_{2}^{\prime 2}+m^{2}}{q_{1}^{\prime 2}+m^{2}}} \\
+\sqrt{\frac{q_{2}^{2}+m^{2}}{q_{1}^{2}+m^{2}}} \frac{1}{\left(\left(\boldsymbol{k}-\boldsymbol{k}^{\prime}\right)^{2}+m^{2}\right)} \sqrt{\frac{q_{1}^{\prime 2}+m^{2}}{q_{2}^{\prime 2}+m^{2}}} \\
-\frac{q^{2}+\frac{N_{C}^{2}+1}{N_{C}^{2}} m^{2}}{\sqrt{q_{1}^{2}+m^{2}} \sqrt{q_{2}^{2}+m^{2}} \sqrt{q_{1}^{\prime 2}+m^{2}} \sqrt{q_{2}^{\prime 2}+m^{2}}}
\end{gathered}
$$

First, we consider the forward direction $\boldsymbol{q}^{2}=0$ and the kernel simplifies to: 


$$
\frac{2 \pi}{\bar{\alpha}} K\left(\boldsymbol{k}, \boldsymbol{k}^{\prime}\right)=\frac{2}{\left(\left(\boldsymbol{k}-\boldsymbol{k}^{\prime}\right)^{2}+m^{2}\right)}-\frac{N_{C}^{2}+1}{N_{C}^{2}} \frac{m^{2}}{\left(\boldsymbol{k}^{\prime 2}+m^{2}\right)\left(\boldsymbol{k}^{2}+m^{2}\right)}
$$

For numerical calculation, we restrict the eigenvalue equation $\widetilde{K} f_{\omega}=\omega f$ to spherically symmetric eigenfunction, and the angular integrations can be done. For the remained integration in $\boldsymbol{k}^{2}$, we use logarithmic variable: $t^{\prime}=\ln k^{2} / \mathrm{m}^{2}$ and we replace the continuous variables by a discrete lattice version for the variables. We introduce the limits: $k_{\min }{ }^{2}=10^{-40}$, $t_{\min }=\ln k_{\min }{ }^{2} / \mathrm{m}^{2}$ and $k_{\operatorname{Max}}{ }^{2}=10^{80}, t_{\operatorname{Max}}=\ln {k_{\operatorname{Max}}}^{2} / \mathrm{m}^{2}$, and dividing the interval [ $t_{\min }, t_{\operatorname{Max}}$ ] into $N_{\text {step }}$ and the lattice space is $\left(t_{\text {Max }}-t_{\text {min }}\right) / N_{\text {step }}$ and we arrive at the discrete vector $f_{n}=f\left(k_{n}\right)$ and the matrix $K_{i, j}=K\left(\boldsymbol{k}_{\boldsymbol{i}}, \boldsymbol{k}_{\boldsymbol{j}}\right)$ :

$$
\int d^{2} \boldsymbol{k}^{\prime} K\left(\boldsymbol{k}, \boldsymbol{k}^{\prime}\right) f\left(k^{\prime}\right)=\int d t^{\prime} K\left(t, t^{\prime}\right) k^{2} f\left(k^{\prime}\right) \rightarrow \sum_{j} K_{i, j} k_{j}{ }^{2} f_{j} .
$$

For $N_{\text {step }}=600$, the numerical first eigenvalues are listed in Table 1 .

Table 1. Numerical result for Higgs mass regulator: the first two Columns are for the eigenvalues, slopes with fixed coupling constant and the last with running-coupling constant.

\begin{tabular}{|c|c|c|c|c|c|}
\hline $\mathrm{n}$ & Energy & Slope & Energy & Slope & Radius \\
\hline 1 & -0.5295 & 0.00000 & -0.4384 & 0.0464 & 3.9 \\
\hline 2 & -0.5268 & 0.00001 & -0.2253 & 0.0144 & $9.1 \times 10^{1}$ \\
\hline 3 & -0.5224 & 0.00003 & -0.1508 & 0.0070 & $2.3 \times 10^{3}$ \\
\hline 4 & -0.5162 & 0.00005 & -0.1131 & 0.0041 & $6.2 \times 10^{4}$ \\
\hline
\end{tabular}

As expected, the spectrum of eigenvalues starts near $E_{B F K L}=-\omega_{B F K L}=-4 \ln 2 \bar{\alpha}_{S}=$ -0.529 with $\bar{\alpha}_{s}=0.2$ and the slopes are very small. The discreteness of the eigenvalues as well as the no vanishing slopes consequence of the lattization: from analytic calculations it is known that the characteristic function leads to a continuous cut in the energy plane beginning at $-4 \ln 2 \bar{\alpha}_{s}=-0.5295$ and extending to $+\infty$.

\section{$4.2 q^{2}$-slopes of the Eigenvalues}

In order to study the $\boldsymbol{q}^{2}$ slopes, we leave the forward direction and we need to consider the $q^{2}$ dependence of the eigenvalues, which is given by:

$$
\omega_{n}\left(\boldsymbol{q}^{2}\right)=\omega_{g}^{0}+\boldsymbol{q}^{2} \frac{\int d^{2} \boldsymbol{k} \int d^{2} \boldsymbol{k}^{\prime} f_{n}\left(\boldsymbol{k}^{\prime 2}\right)\left[K^{1}\left(\boldsymbol{k}, \boldsymbol{k}^{\prime}\right)+2 \delta^{2}\left(\boldsymbol{k}-\boldsymbol{k}^{\prime}\right) \omega_{g}^{1}\left(\boldsymbol{k}^{2}\right)\right] f_{n}\left(\boldsymbol{k}^{2}\right)}{\int d^{2} k^{\prime}\left|f_{n}\left(\boldsymbol{k}^{\prime 2}\right)\right|^{2}}
$$

where $\omega_{g}^{0}$ are the eigenvalues of the forward kernel $K$ (see eq. (14)), $f_{n}\left(\boldsymbol{k}^{2}\right)$ the corresponding (rotationally invariant) eigenfunctions, and $K^{1}, \omega_{g}^{1}$ the corrections of the order $\boldsymbol{q}^{2}$ to the forward BFKL kernel and the gluon trajectory, resp. After straightforward calculation, the numerical result for the slope are in the second column of the Table 1.

\subsection{Running coupling corrections}

Let us now to consider effect of the running coupling constant. In our numerical analysis, we follow the conventions used in [22] and consider the running coupling constant given by:

$$
\alpha_{s}\left(q^{2}\right)=\frac{3.41}{\beta_{0}\left[\ln \left(q^{2}+\mathrm{R}_{0}^{2}\right)+\ln \left(m^{2} / \Lambda_{Q C D}\right)^{2}\right.},
$$


where $R_{0}$ and the momenta $q^{2}$ are defined in units of the regulator mass $m=m_{h}=0.54 \mathrm{GeV}$. A symmetrized definition for the kernel with running coupling is given by:

$$
\alpha_{s}\left(k^{2}\right) K\left(\boldsymbol{k}, \boldsymbol{k}^{\prime}\right) \rightarrow \sqrt{\alpha_{s}\left(k^{2}\right)} K\left(\boldsymbol{k}, \boldsymbol{k}^{\prime}\right) \sqrt{\alpha_{s}\left(k^{\prime 2}\right)} .
$$

For the slope, we have to leave the forward direction and in addition to the $q^{2}$ expansion of the kernel and of the trajectory function described previously, we also need the expansion of the running coupling. After to consider all these contributions, we can obtain numerical results for the eigenvalues and slope in the Table 2, (see the third and fourth columns). In Fig 4 , we show the shape of the eigenfunction. In order to have a measure for the extension of the wave functions into the region of large momenta, we define a logarithmic radius:

$$
r_{n}=<\ln \left(q^{2}\right)>=\frac{\int d^{2} k^{\prime}\left|f_{n}\left(k^{\prime 2}\right)\right|^{2} \ln \left(q^{2}\right)}{\int d^{2} k^{\prime}\left|f_{n}\left(k^{\prime 2}\right)\right|^{2}}
$$

where momenta are in units of $m_{h}=0.45 \mathrm{GeV}$. Our analysis shows that, again for $\mathrm{n}<18, r_{n}$ grows linearly with $n: r_{n} \approx-2.7+6.6 n$. Clearly, with increasing $n$ the support of the wave function quickly becomes dominant by the UV region, only $n=1$ is concentrate in the soft region.

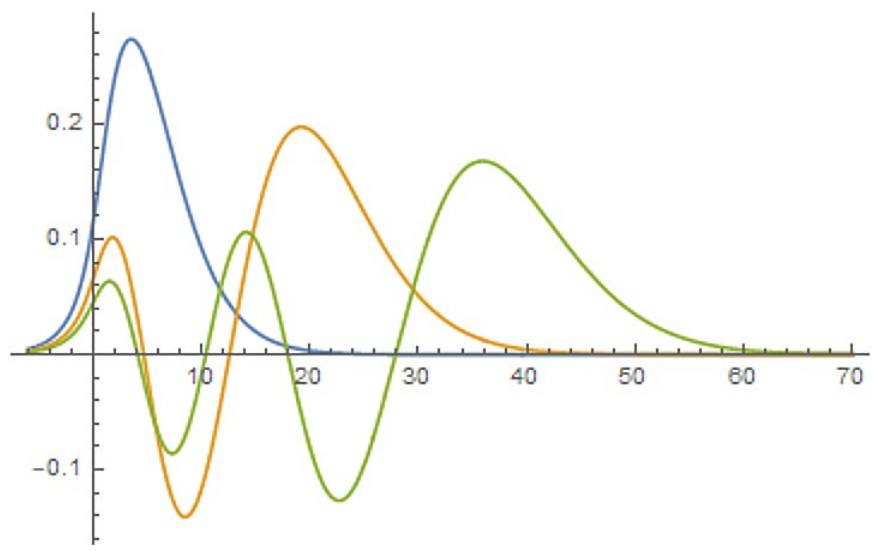

Fig. 4. Three leading wave function $(\mathrm{n}=1,3,5)$ as a function of $\ln q^{2}$ with running coupling constant and Higgs mass regulator.

\subsection{Numerical study for the Wilsonian optimized regulator}

We repeat the analysis described above considering the ERG regulator introduced previously. This numerical analysis is more involved. In Table 2 we present our results, after the symmetrization of the kernel, the numerical integral over the angle between the vectors $\left(\boldsymbol{k}, \boldsymbol{k}^{\prime}\right)$, the expansion in power of $q^{2}$, first for the fixed coupling constant (first and second row) and then for the running coupling.

Table 2. Numerical result for Wilsonian regulator with IR cutoff $k=1.0$ : the first two Columns are for the eigenvalues, slopes with fixed coupling constant and the last with running-coupling constant.

\begin{tabular}{|c|c|c|c|c|c|}
\hline $\mathrm{n}$ & Energy & Slope & Energy & Slope & Radius \\
\hline 1 & -0.5295 & 0.00002 & -0.43 & 0.034 & 4.7 \\
\hline 2 & -0.5269 & 0.00009 & -0.22 & 0.0091 & $1.2 \times 10^{2}$ \\
\hline 3 & -0.5225 & 0.00019 & -0.15 & 0.0042 & $3.3 \times 10^{3}$ \\
\hline 4 & -0.5165 & 0.00033 & -0.11 & 0.0024 & $8.9 \times 10^{4}$ \\
\hline
\end{tabular}


Comparison with the Higgs mass regulator and fixed coupling constant listed in Table 1 we show very small differences in the spectrum of eigenvalues. Slope are still small and differ those of the Higgs mass regulator.

For the running coupling constant, we show the result in the Table 3 , for different values of the Infrared cutoff (in GeV)

Table 3. Numerical results for eigenvalues, slopes and radii, with running-coupling constant and for different values of the IR cutoff $k$

\begin{tabular}{|c|ccc|cccc|ccc|}
\hline & \multicolumn{3}{|c|}{ Energy } & \multicolumn{3}{c|}{ Slope } & \multicolumn{3}{c|}{ Radius } \\
\hline $\mathrm{n}$ & $\mathrm{k}=0.54$ & $\mathrm{k}=1.0$ & $\mathrm{k}=5.0$ & $\mathrm{k}=0.54$ & $\mathrm{k}=1.0$ & $\mathrm{k}=5.0$ & $\mathrm{k}=0.54$ & $\mathrm{k}=1.0$ & $\mathrm{k}=5.0$ \\
\hline 1 & -0.53 & -0.43 & -0.29 & 0.17 & 0.034 & 0.0007 & 2.2 & 4.7 & $3.4 \times 10^{1}$ \\
\hline 2 & -0.25 & -0.22 & -0.17 & 0.04 & 0.009 & 0.0002 & $5.2 \times 10^{1}$ & $1.2 \times 10^{2}$ & $1.0 \times 10^{3}$ \\
\hline 3 & -0.16 & -0.15 & -0.12 & 0.02 & 0.004 & 0.0001 & $1.4 \times 10^{3}$ & $3.3 \times 10^{3}$ & $2.8 \times 10^{4}$ \\
\hline 4 & -0.12 & -0.11 & -0.10 & 0.01 & 0.002 & 0.00007 & $3.7 \times 10^{4}$ & $8.9 \times 10^{4}$ & $7.8 \times 10^{5}$ \\
\hline
\end{tabular}

A qualitative discussion above our results which are dependent on the cutoff parameter $\mathrm{k}$ : with increasing cutoff, the intercepts as well as the slope become smaller, whereas the radii grow. Finally, one can observe that for the inverse values of the Energy lie on a straight lines and the inverse of the slope increase proportional to $n^{2}$. It is interesting to note that similar results are reported in ref. [29]. The shape of the wave function is showed in Fig 5.
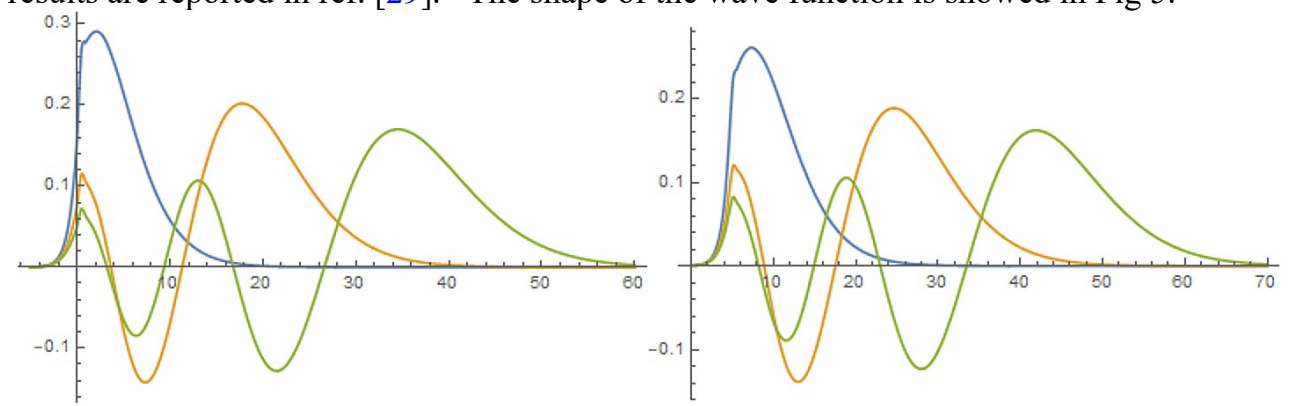

Fig. 5. Three leading wave function $(n=1,3,5)$ for the scale $k=0.54$ and $5 \cdot 0 \mathrm{GeV}$ as a function of $\ln q^{2}$.

For each scale we observe that the nonleading eigenfunctions have their support at large momenta, in agreement with the behaviour of the radii listen in the tables.

\section{Conclusion}

In summary, we found a formulation of the BFKL Pomeron with a IR regulated Wilsonian cutoff. Conceptually the Pomeron fields appears as a bound state of two reggeized gluons. As a first step in this program, we have investigated for different numerical values of the $k$ cutoff. Our numerical values exhibit important features which will play a crucial role in future analysis: the intercepts and the $q^{2}$ slopes of the bound state poles decrease and go to zero, once we move from the leading eigenvalues down towards the accumulation point at $\omega=0$. Only the eigenfunction of the leading trajectory is typically 'soft', i.e. it has support in the small-momentum region. The other nonleading eigenfunctions extend more and more into the UV region, i.e. they become 'hard'. 
As we have already said, these results, in the next step of our program, can be used to define the (local) Pomeron fields which serve as input to the RFT. For this, we will need to consider the study of the three Pomeron vertex, which can be obtained by projecting the 2 to 4 reggeized gluon vertex onto the Pomerons states [34-35]. Finally, its infrared behavior will have to be matched to the results of our previous papers devoted to the IR behavior.

Acknowledgements: $\mathrm{CCH}$ is grateful to the ISMD organizers for kind invitation, warm hospitality and this research was supported in part by the FONDECYT grant $\mathrm{N}^{\circ} 1180118$ and the DGIIP USM.

\section{References}

[1] J. Bartels, C. Contreras and G. P. Vacca, JHEP 1603201 (2016)

[2] J. Bartels, C. Contreras and G. P. Vacca, Phys. Rev. D 95 no.1, 014013 (2017)

[3] J. Bartels, C. Contreras and G. P. Vacca, JHEP (2018) (to be published)

[4] C. Wetterich, Phys. Lett. B 30190 (1993)

[5] T. R. Morris, Phys. Lett. B 329241 (1994)

[6] V. N. Gribov and A. A. Migdal, Sov. Phys. JETP 28784 (1969)

[7] V. N. Gribov and A. A. Migdal, Sov. J. Nucl. Phys. 8583 (1969)

[8] H. D. Abarbanel and J.B. Bronzan, Phys. Rev. D 902397 (1974)

[9] A. A. Migdal, A. M. Polyakov, and K. A. Ter-Martirosian, Phys. Lett. B 48239 (1974)

[10] L. N. Lipatov, Nucl. Phys. B 452369 (1995)

[11] L. N. Lipatov, Sov. J. Nucl. Phys. 23338 (1976)

[12] E. A. Kuraev, L. N. Lipatov and V. S. Fadin, Sov. Phys. JETP 44443 (1976)

[13] E. A. Kuraev, L. N. Lipatov and V. S. Fadin, Sov. Phys. JETP 45199 (1977)

[14] I. I. Balitsky and L. N. Lipatov, Sov. J. Nucl. Phys. 28822 (1978)

[15] J. Bartels and M. Wusthoff, Z. Phys. C 66157 (1995)

[16] J. Bartels, L. N. Lipatov and M. Wusthoff, Nucl. Phys. B 464 (1996)

[17] M. A. Braun and G. P. Vacca, Eur. Phys. J. C 6147 (1999)

[18] J. Bartels, M. G. Ryskin and G. P. Vacca, Eur. Phys. J. C 27101 (2003)

[19] J. Bartels, L. N. Lipatov and G. P. Vacca, Nucl. Phys. B 706 (2005)

[20] J. Bartels, L. N. Lipatov and G. P. Vacca, Phys. Lett. B 477178 (2000)

[21] R. A. Janik and J. Wosiek, Phys. Rev. Lett. 821092 (1999)

[22] L. N. Lipatov, Sov. Phys. JETP 63904 (1986)

[23] M. Braun, G. P. Vacca and G. Venturi, Phys. Lett. B 388823 (1996)

[24] E. Levin, L. N. Lipatov and M. Siddikov, Phys. Rev. D 94 no.9, 096004 (2016)

[25] E. Levin, L. N. Lipatov and M. Siddikov, Eur. Phys. J. C 75 no.11, 558 (2015)

[26] E. Levin, L. N. Lipatov and M. Siddikov, Phys. Rev. D 89 no.7, 074002 (2014)

[27] H. Kowalski, L. N. Lipatov, D. A. Ross and O. Schulz, Eur. Phys. J. C 77 no.11, 777 (2017)

[28] H. Kowalski, L. N. Lipatov and D. A. Ross, Eur. Phys. J. C 76 no.1, 23 (2016)

[29] H. Kowalski, L. N. Lipatov and D. Ross, Eur. Phys. J. C 74 no.6, 2919 (2014)

[30] R. Kirschner and L. N. Lipatov, Sov. Phys. JETP 56266 (1982)

[31] R. Kirschner and L. N. Lipatov, Phys. Rev. D 26 (12021982)

[32] R. Kirschner and L. N. Lipatov, Nucl. Phys. B 213122 (1983)

[33] D. F. Litim, Phys. Rev. D 64105007 (2001)

[34] J. Bartels, Nucl. Phys. B 175365 (1980)

[35] J. Kwiecinski and M. Praszalowicz, Phys. Lett. B 94413 (1980) 